\title{
June 2017 Pulmonary Case of the Month
}

Robert Horsley, MD

Lewis J. Wesselius, MD

Department of Pulmonary Medicine

Mayo Clinic Arizona

Scottsdale, AZ USA

\section{History of Present IIIness}

A 61-year-old woman presented to the emergency department for 3 days of fevers up to $102^{\circ} \mathrm{F}$, malaise, and progressive shortness of breath. Her symptoms started immediately after he last naltrexone injection for alcohol use disorder.

\section{Past Medical History, Social History and Family History}

- Alcohol use disorder

- Treated with monthly naltrexone injections, received 3 doses total, and gabapentin

- No other previous medical issues

- Nonsmoker

\section{Physical Examination}

- Vital signs: Pulse 100, BP 108/90, respiratory rate 34, SpO2 93\% 10L nonrebreathing mask

- Cyanotic on room air

- Lungs clear

\section{Radiography}

A portable chest x-ray was performed in the emergency department (Figure 1).

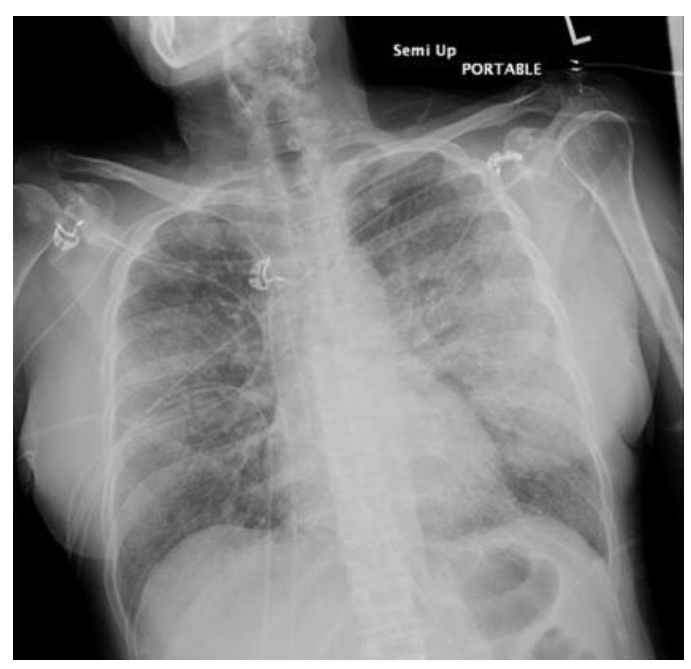

Figure 1. AP chest radiograph taken in the emergency department. 
A thoracic CT scan was performed (Figure 2).

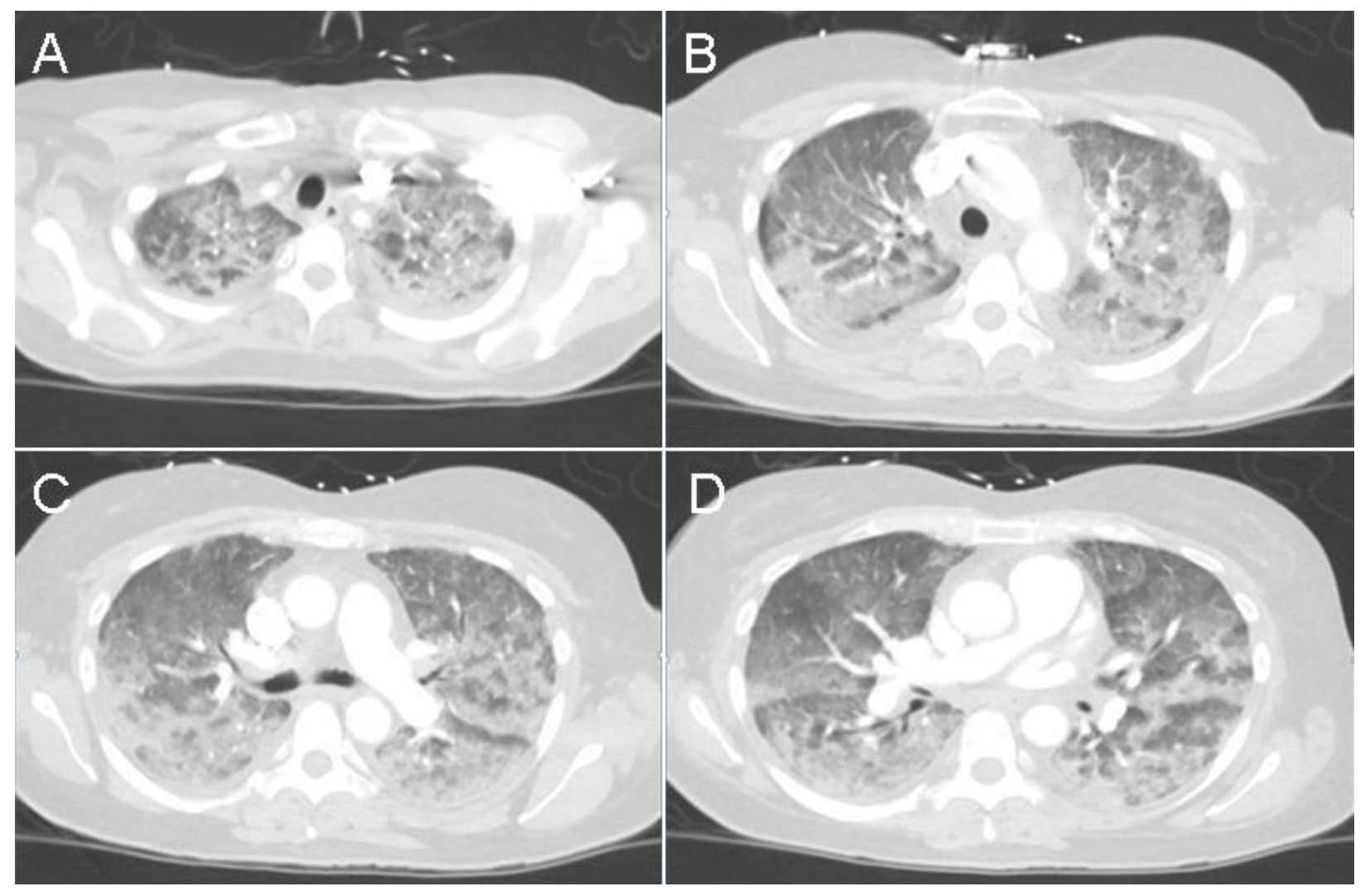

Figure 2. Representative images from thoracic CT in lung windows.

\section{Laboratory}

- $\quad$ CBC showed a white blood cell count of 12,000 cells $/ \mathrm{mcL}$.

- The differential shows a left shift.

- Lactate was $5.2 \mathrm{mmol} / \mathrm{L}$.

Which of the following is (are) true?

1. A lactate level of 5.2 can be a normal finding in a critically ill patient

2. Her symptoms are likely an allergic reaction to naltrexone

3. The most likely diagnosis is an atypical pneumonia

4. 1 and 3

5. All of the above 


\section{Correct! \\ 3. The most likely diagnosis is an atypical pneumonia}

Naltrexone, an opioid antagonist, is used to treat alcoholism and opioid dependence (1). A diffuse pneumonia has been rarely reported secondary to naltrexone (1). A markedly elevated lactate is usually an ominous finding (2). The normal blood lactate concentration in unstressed patients is $0.5-1 \mathrm{mmol} / \mathrm{L}$. Patients with critical illness can be considered to have normal lactate concentrations of less than $2 \mathrm{mmol} / \mathrm{L}$. Lactate levels above $4 \mathrm{mmol}$ are associated with major metabolic dysregulation, tissue hypoperfusion, the effects of certain drugs or toxins, and congenital abnormalities in carbohydrate metabolism.

Her chest x-ray and CT scan both show diffuse consolidation consistent with an atypical pneumonia. This term usually refers to pneumonia caused by certain bacteria, including Legionella pneumophila, Mycoplasma pneumoniae, Chlamydophila pneumoniae and Chlamydia psittaci (3). It is called "atypical" because the symptoms differ from those of pneumonia due to other common bacteria. Even though it is called atypical, these infections are not uncommon. Influenza is sometime lumped into this group although it is caused a virus. Radiographic findings in atypical pneumonia are often considerably more impressive than the clinical findings.

She was given a dose of $125 \mathrm{mg}$ of methylprednisolone in the emergency department and blood cultures were obtained. She was admitted to the intensive care unit and intubated. Treatment with norepinephrine and dobutamine along with oseltamivir, ceftriaxone, and levofloxacin was begun.

The next morning, she is clinically unchanged although her fever is decreased and her lactate has decreased below $2 \mathrm{mmol}$. Her blood cultures showed no growth and respiratory viral and bacterial pathogen panels were negative.

What should be done next?

1. Begin therapy for coccidioidomycosis

2. Bronchoscopy with bronchoalveolar lavage

3. Continue present therapy since she seems to be responding

4. 1 and 3

5. All of the above 


\section{Correct!}

\section{Bronchoscopy with bronchoalveolar lavage}

Really any of the choices are acceptable. She does seem somewhat clinically better although no diagnosis is yet established. Valley Fever or coccidioidomycosis is almost always a consideration in Arizona. Our choice was to perform a bronchoscopy with bronchoalveolar lavage since she was intubated making it relatively uncomplicated and potentially diagnostic.

Her airways were unremarkable. Bronchoscopy with bronchoalveolar lavage was performed. Cultures were ordered and below is her bronchoalveolar lavage differential (Figure 3).

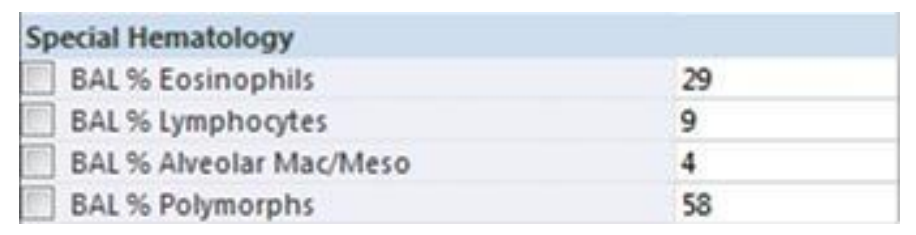

Figure 3. Bronchoalveolar lavage (BAL) differential.

Which of the following are true regarding the BAL differential?

1. A high percentage of BAL eosinophils is inconsistent with coccidioidomycosis

2. A high percentage of eosinophils is consistent with acute eosinophilic pneumonia

3. A normal percentage of blood eosinophils is inconsistent with acute eosinophilic pneumonia

4. 1 and 3

5. All of the above 


\section{Correct! \\ 2. The high percentage of eosinophils is consistent with acute eosinophilic pneumonia}

The percentage of BAL eosinophils is quite high. Although this varies from laboratory to laboratory it is generally less than $5 \%$. Eosinophilia can be seen in both the BAL and serum in coccidioidomycosis although the percentage in our patient is higher than usually seen in coccidioidomycosis which averages about $8 \%$ (4). BAL eosinophilia is seen, often over $50 \%$, in acute eosinophilic pneumonia (5). In contrast to chronic eosinophilic pneumonia, the peripheral eosinophil count is usually no elevated in acute eosinophilic pneumonia.

Which of the following are indicated next?

1. Begin treatment with high dose corticosteroids

2. Carefully inspect the BAL fluid for Strongyloides

3. Obtain serum $\lg \mathrm{E}$

4. 1 and 3

5. All of the above 


\section{Correct! \\ 5. All of the above}

A number of infectious and noninfectious causes can result in eosinophilic pneumonia (5). Most of the infectious causes are parasites and include ascaris, hookworm, schistosomiasis, and strongyloidiasis. The later can result in serious, potentially fatal pulmonary infections in the context of the hyperinfection syndrome, resulting from heavy parasite burdens. This is most often seen in immunocompromised hosts with deficiencies in cell-mediated immunity.

The noninfectious causes include an allergic reaction, exposures to a drug or toxin and a number of idiopathic causes (chronic eosinophilic pneumonia, acute eosinophilic pneumonia, Churg-Strauss syndrome, hypereosinophilic syndrome). The most likely diagnosis appears to be acute eosinophilic pneumonia. However, allergic bronchopulmonary aspergillosis (ABPA) can closely mimic acute eosinophilic pneumonia. The IgE is usually markedly elevated in ABPA, and practically speaking, a normal IgE excludes the diagnosis. Our patient's IgE was normal.

We identified 2 other case reports of acute eosinophilic pneumonia following naltrexone injection $(1,6)$. We speculate that the dose of methylprednisolone given in the emergency department lowered the eosinophil percentage in her BAL to slightly below what is usually seen in acute eosinophilic pneumonia. Our patient was continued on corticosteroids and her clinical findings and chest x-ray rapidly improved (Figure 4).

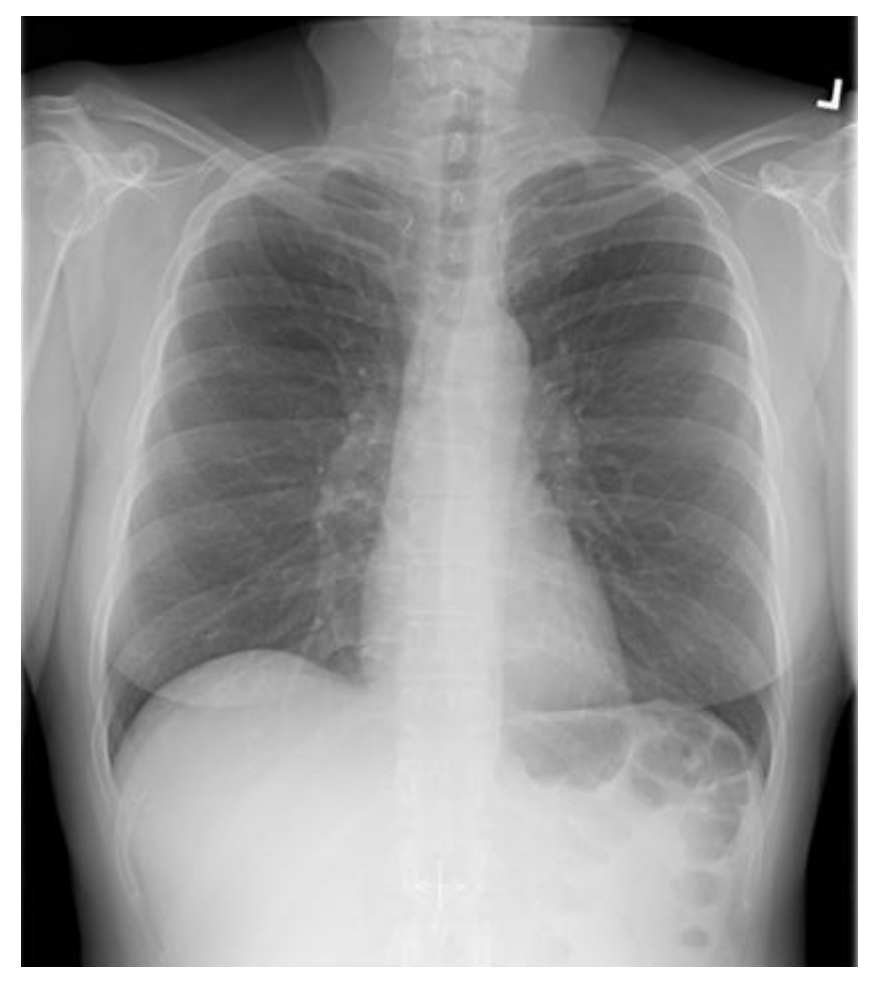

Figure 4. Follow-up PA chest radiograph. 


\section{References}

1. Garbutt JC, Kranzler HR, O'Malley SS, Gastfriend DR, Pettinati HM, Silverman BL, Loewy JW, Ehrich EW; Vivitrex Study Group. Efficacy and tolerability of long-acting injectable naltrexone for alcohol dependence: a randomized controlled trial. JAMA. 2005 Apr 6;293(13):1617-25. [CrossRef] [PubMed]

2. Gunnerson KJ. Lactic acidosis. Medscape. March 7, 2017. Available at: http://emedicine.medscape.com/article/167027-overview (accessed 5/30/17).

3. CDC. Atyical pneumonia. July 14, 2016. Available at: https://www.cdc.gov/pneumonia/atypical/index.html (accessed 5/30/17).

4. Alzoubaidi MSS, Knox KS , Wolk DM, Nesbit LA, Jahan K, Luraschi-Monjagatta C. Eosinophilia in coccididiomycosis. Am J Resp Crit Care Med. 2013;187:A5573 [Abstract].

5. Akuthota P, Weller PF. Eosinophilic pneumonias. Clin Microbiol Rev. 2012 Oct;25(4):649-60. [CrossRef] [PubMed]

6. Kim H, Ali M, Buch K. Eosinophilic Pneumonia Induced by Injectable Naltrexone. Am J Respir Crit Care Med. 2014;189:A2283 [Abstract]. 